EPJ Web of Conferences 16, 03007 (2011)

DOI: $10.1051 /$ epjconf/20111603007

(C) Owned by the authors, published by EDP Sciences, 2011

\title{
Direct imaging and new technologies to search for substellar companions around MGs cool dwarfs
}

\author{
M.C. Gálvez-Ortiz ${ }^{1, a}$, J.R.A. Clarke ${ }^{1}$, D.J. Pinfield ${ }^{1}$, S.L. Folkes ${ }^{1}$, J.S. Jenkins ${ }^{2}$, \\ A.E. García Pérez ${ }^{1}$, B. Burningham ${ }^{1}$, A.C. Day-Jones ${ }^{2,1}$ and H.R.A. Jones ${ }^{1}$ \\ ${ }^{1}$ Centre for Astrophysics Research, Science and Technology Research Institute, \\ University of Hertfordshire, Hatfield AL10 9AB, UK \\ ${ }^{2}$ Department of Astronomy, Universidad de Chile, Casilla Postal 36D, Santiago, Chile
}

\begin{abstract}
We describe here our project based in a search for sub-stellar companions (brown dwarfs and exo-planets) around young ultra-cool dwarfs (UCDs) and characterise their properties. We will use current and future technology (high contrast imaging, high-precision Doppler determinations) from the ground and space (VLT, ELT and JWST), to find companions to young objects. Members of young moving groups (MGs) have clear advantages in this field. We compiled a catalogue of young UCD objects and studied their membership to five known young moving groups: Local Association (Pleiades moving group, 20-150 Myr), Ursa Mayor group (Sirius supercluster, $300 \mathrm{Myr}$ ), Hyades supercluster (600 Myr), IC 2391 supercluster (35 Myr) and Castor moving group (200 Myr). To assess them as members we used different kinematic and spectroscopic criteria.
\end{abstract}

\section{INTRODUCTION}

Why adaptive optics (AO) Imaging of UCD in MGs? Nearby MG members will make ideal targets for AO searches seeking faint companions. Since substellar companions cool and fade with time, target young systems provide higher flux ratios and so higher probability of detection compared to typical older field objects. With well constrained age, composition and distances, our MG members would provide an ideal population, benchmark object, to test atmosphere and evolutionary UCD models.

The sample was selected from photometric and astrometric criteria in order to search for UCDs in MGs. Our final red object catalogue was made up of 817 objects. We could conclude that 132 of these objects were possible members of a MG. The detailed selection procedure can be found in [1].

\section{KINEMATIC AND YOUTH CONSTRAINTS (ROTATIONAL VELOCITIES)}

We computed the galactic space velocity components $(\mathrm{U}, \mathrm{V}, \mathrm{W})$ of the sample taking into account their possible membership to one or several MGs, and plotted them (see Fig. 1 Left side) in the UV and WV planes including the boundaries (solid line) that delimit the young disk population as defined by [2]. We then applied a simple criterion that consists of identifying a possible member of one of the five MGs by its relative position in the UV and VW diagrams with respect to the boxes (dashed lines) that marked the velocity ranges of different MGs. The boxes are defined by studies with higher mass member samples from the literature ([3]; [4]). The centre of the five main MGs are marked with

ae-mail: M.Galvez-Ortiz@herts.ac.uk

This is an Open Access article distributed under the terms of the Creative Commons Attribution-Noncommercial License 3.0, which permits unrestricted use, distribution, and reproduction in any noncommercial medium, provided the original work is properly cited. 

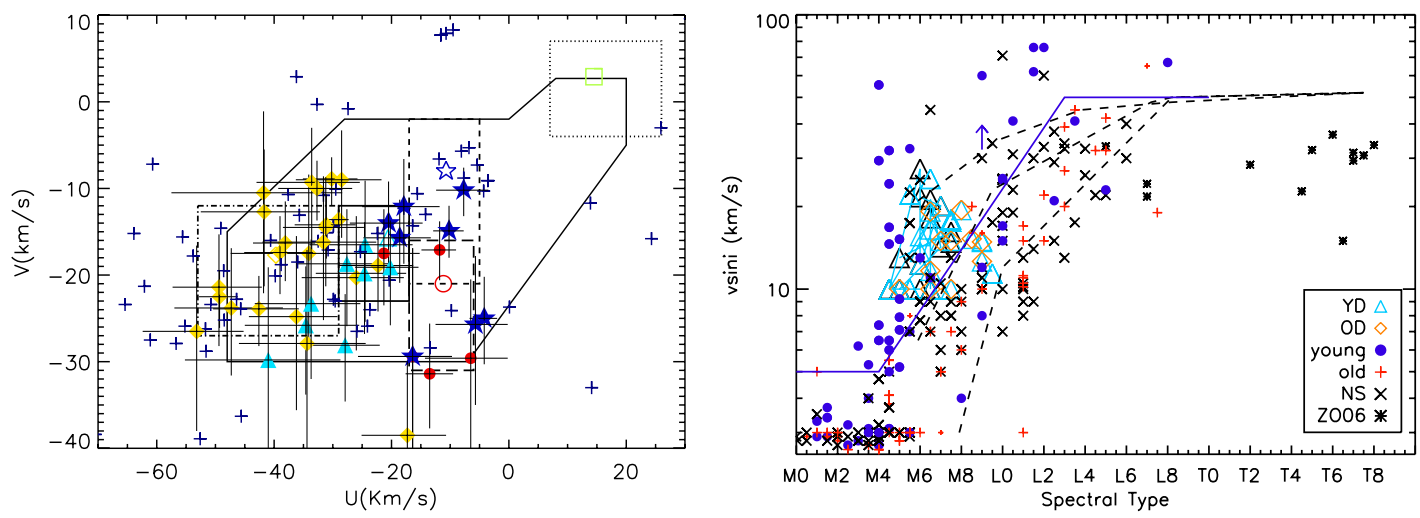

Figure 1. Left: UV space motion diagrams for our sample. The young disk boundaries defined by [2] are also plot in continuous line. See Section 2 for explanation. Right: Spectral type versus $v \sin i$. See Section 3 for explanation.

large open symbols (squares for Sirius, diamonds for Hyades, triangles for IC 2391, circles for Pleiades and stars for Castor) and our kinematic members are shown as the corresponding filled symbols. If an object does not lie inside one of the MGs boxes but is inside the young disk area, it is classified as a young disk (YD). We find 49 kinematic YD objects with 35 of them belonging to one of the five MGs.

It is known that there is contamination of old objects that share kinematic properties with the genuine young MG members. To further assess the membership of our kinematic candidates, we will combine several methods (lithium I doublet at $6708 \AA$, activity/age relation, rotational velocities $(v \sin i)$, etc) to provide robust age constraints. For further details see [1].

We study here the relation between age and $v \sin i$ in order to use the last one to constrain the age of our sample. Several studies have proved that after becoming a fully convective object (spectral types $>$ M3), when gravitational contraction is finished, rotational braking is present in all $\mathrm{M}$ type objects. The spin-down times are longer for Late $\mathrm{M}$ dwarfs than for earlier M types (e.g. [5]; [6] etc). Following these studies, we used $v \sin i$ to differentiate between young and older M type UCD populations. We measure the $v \sin i$ of 55 of the total 69 sample, and of 40 of the total 45 YD objects and plot them (see Fig. 1 Right side) in a $v \sin i$-spectral type diagram from [5]. Young and old populations occupy distinct regions in the diagram. Circles are supposed to be young, pluses old and crosses have unknown age. Asterisks come from [6]. Our data sample is plotted in different symbols depending on whether they have been classified as YD or as old disk (OD). We also mark the ages of 2, 5 and $10 \mathrm{Gyr}$ (from upper left to lower right) as dashed lines and the "apparent" separation between the considered "young" and "old" objects as a solid line, that we used as a criterion for determining the possible membership of our targets to the correspondent MG. From the 40 kinematic YD candidates, 31 present a projected $v \sin i$ in agreement with our criteria of youth and 11 that can not be dismissed from $v \sin i$ information.

\section{AO IMAGING}

The kinematic confirmed members will provide a substantial sample of ideal targets for AO imaging.

Taking currect technology capabilityes, like NACO/SDI in VLT (operating with a laser guide star, which could reach photon noise limits for a brightness ratio of 11 magnitudes in the H-band at $0.1^{\prime \prime}$ separation), our youngest and closest MG members (in the IC 2391 or Pleiades MGs) would allow AO to probe to $\approx 1 \mathrm{M}_{\text {Jup }}$ at separations of $\approx 1 \mathrm{AU}$. 
Research, Science and Technology of Brown Dwarfs and Exoplanets

\section{References}

[1] J.R.A Clarke, D.J. Pinfield, M.C. Gálvez-Ortiz, et al., MNRAS, accepted, arXiv:0805.4772, (2009)

[2] O.G. Eggen, PASP, 101, (1989) 399

[3] D. Montes, J. López-Santiago, M.C. Gálvez, et al., MNRAS, 328, (2001) 45

[4] D. Barrado Y Navacués, A\&A, 339, (1998) 891B

[5] A. Reiner \& G. Basri, AJ, 684 (2008) 1390

[6] M.R. Zapatero-osorio, E.L. Martín, H. Bouy, et al., Apj, 647 (2006) 1405 